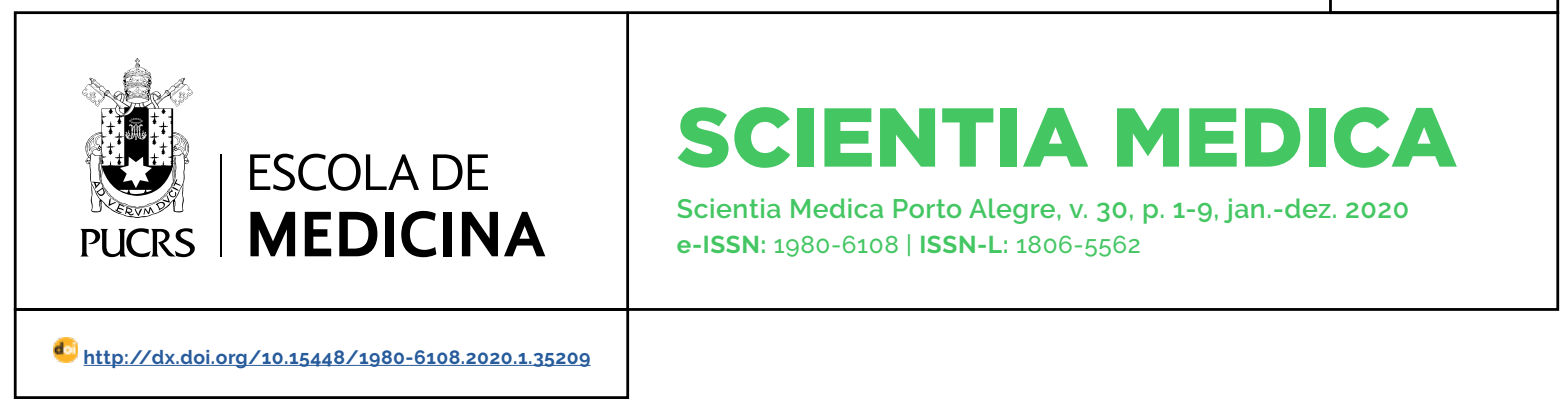

EDUCAÇÃO EM CIÊNCIAS DA SAÚDE

\title{
Avaliação dos graus de ansiedade em acadêmicos de uma faculdade privada
}

\author{
Evaluation of the degree of anxiety in academics of a private college
}

\section{Evlin Spósito Arruda ${ }^{1}$}

orcid.org/0000-0003-3339-9284 evlin.sposito@hotmail.com

\author{
Nadielle Silva Bidu ${ }^{2}$ \\ orcid.org/0000-0001-9793-1044 \\ nadiellebidu@hotmail.com
}

\section{Rodrigo Santos \\ Damasceno ${ }^{1}$}

orcid.org/0000-0002-0527-6183

rodrigodamascena@fainor.com.br

\section{Rafael Luiz de Araújo Rodrigues $^{1}$ \\ orcid.org/0000-0002-5686-8795 \\ faelfarmac@hotmail.com}

Recebido em: 3 ago. 2019. Aprovado em: 20 abr. 2020. Publicado em: 24 jul. 2020.

\section{Resumo}

Objetivo: este estudo teve como objetivo avaliar o grau de ansiedade dos acadêmicos de uma instituição de ensino superior privada.

Métodos: foi realizada uma pesquisa de corte transversal com abordagem descritiva, quantitativa e observacional em 366 alunos aleatoriamente escolhidos que estavam matriculados no primeiro semestre do ano de 2019. A coleta de dados foi realizada por meio de um questionário com questões sociodemográficas-comportamentais e questões do Inventário de Ansiedade Beck. Os graus de ansiedade (mínimo ou ausente, leve, moderado e grave) foram determinados pelo somatório dos escores do inventário de Beck.

Resultados: do total da amostra, 62,3\% apresentou algum grau de ansiedade, sendo os cursos da área de saúde os que mais apresentaram estudantes com graus de ansiedade. O sexo e a idade foram as variáveis que apresentaram correlação significativa com os graus de ansiedade.

Conclusão: este estudo alerta sobre a ocorrência de transtornos de ansiedade em estudantes universitários, o que implica em uma necessidade crescente de estratégias, dentro do ambiente acadêmico, para reverter e evitar o desencadeamento de patologias de saúde mental nos universitários.

Palavras-chave: ansiedade, saúde mental, universitários.

\section{Abstract}

Aims: this study aimed to evaluate the degree of anxiety of academics from a private higher education institution.

Methods: a cross-sectional, descriptive, quantitative and observational study was conducted in 366 randomly selected students enrolled in the first semester of 2019. Data collection was performed through a questionnaire with sociodemographic-behavioral questions and questions of the Beck Anxiety Inventory. The degrees of anxiety (minimal or absent, mild, moderate, and severe) were determined by the sum of Beck's inventory scores.

Results: of the total sample, $62.3 \%$ presented some degree of anxiety, and the courses in the health area presented the most students with degrees of anxiety. Sex and age were the variables that showed a significant correlation with the degrees of anxiety.

Conclusion: this study warns about the occurrence of anxiety disorders in university students, which implies a growing necessity for strategies within the academic environment to revert or prevent the onset of mental health pathologies in university students.

Keywords: anxiety, mental health, college students 


\section{Introdução}

Ansiedade é uma resposta emocional adaptativa a incertezas ou situações alarmantes que nos força a agir e a encontrar uma resposta apropriada para lidar com os sentimentos. Os transtornos de ansiedade encontram-se entre as alterações psiquiátricas mais comumente diagnosticados. Ocasionados por pressões de uma sociedade moderna, tecnológica e principalmente cada vez mais competitiva, o aparecimento dos distúrbios de ansiedade ocorre geralmente no início da vida adulta do individuo, na qual há um exagerado estado de excitação, sensação de temor, incerteza ou apreensão (1-3).

As transformações maturacionais que ocorrem na transição entre as fases adolescente e adultos, preocupações com o futuro, expectativas familiares, cobranças pessoais e a conquista de uma meta já programada são elementos que colaboram extraordinariamente para o aparecimento da ansiedade em jovens. Nesse cenário, os universitários têm sido largamente observados e têm apresentado niveis abundantes de ansiedade, estresse e depressão. A detecção e o tratamento nesta fase da vida são primordiais para se evitar danos à saúde mental e promover o bem-estar fisico, psicológico e profissional $(1,3)$.

Nos indivíduos universitários, o transtorno de ansiedade é a patologia de saúde mental mais frequentemente observada. Os sintomas psicológicos mais comuns incluem pânico, nervosismo antes da aula, incapacidade de realizar trabalhos acadêmicos, desatenção no momento de uma avaliação, ou falta de interesse em alguma disciplina dificil. Enquanto os sintomas fisiológicos denotam nervosismo, palmas das mãos frias e suadas, aceleração dos batimentos cardiacos e da frequência respiratória, ou dor no estômago $(2,4,5)$.

Para analisar os niveis de ansiedade pode-se utilizar o Beck Anxiety Inventory (BAI), aqui denominado de escala BAl, que é um autorrelato de 21 itens que classifica os sintomas do transtorno de ansiedade. Os participantes indicam o grau em que eles experimentaram cada sintoma listado nas últimas duas semanas em uma escala de quatro possiveis respostas, que varia de 0 (nada) a 3 (severamente). O resultado é dado em uma escala de pontos, com categorizações especificas: de o a 7: grau mínimo de ansiedade; de 8 a 15: ansiedade leve; de 16 a 25 ansiedade moderada; de 26 a 63: ansiedade grave, sendo o resultado de 63 pontos o mais elevado, demonstrou que a escala BAI possui fortes propriedades psicométricas (3, 4,6-8).

Sendo assim, este artigo busca avaliar o grau de ansiedade dos acadêmicos de uma instituição de ensino superior privada.

\section{Métodos}

Pesquisa do tipo corte transversal com abordagem descritiva, quantitativa e observacional, realizada em uma faculdade particular localizada na cidade de Vitória da Conquista, BA. Utilizou-se uma amostragem probabilística sistemática, com cálculo amostral com abordagem bayesiana, sendo o conjunto amostral constituindo-se por 366 estudantes universitários devidamente matriculados no primeiro semestre do ano de 2019.

Os critérios de inclusão compreenderam alunos matriculados no período 2019.1, presentes na área interna da faculdade no momento da coleta de dados, escolhidos de forma aleatória e que se disponibilizam a responder o questionário. Não foram incluidos na pesquisa todos aqueles que não atendiam aos critérios de inclusão previamente definidos e todos aqueles que não consentiram em participar do estudo. A coleta de dados ocorreu do periodo de fevereiro a março de 2019.

A pesquisa foi realizada através de um questionário com sete questões sociodemográfico-comportamentais elaboradas pelos pesquisadores, além das 21 questões da escala BAI adaptadas e padronizadas para a realidade brasileira referentes à avaliação dos sintomas de ansiedade. O questionário contempla variáveis como sexo, idade, estado civil, moradia, se possui filhos, curso universitário matriculado e atividade de lazer praticada.

Os cursos dos participantes da pesquisa foram categorizados por área do conhecimento, sendo área de saúde (enfermagem, farmácia, fisioterapia, odontologia e estética e cosmética), área de exatas (ciências contábeis, ciências da computa- 
ção, engenharia civil, engenharia de produção e engenharia elétrica) e área de humanas (direito, administração, pedagogia, arquitetura, psicologia, design de moda e design de interior).

Os escores individuais obtidos a partir da escala BAI foram somados e os pontos de corte posteriormente adotados foram: variação de 0 a 7. mínimo; variação de 8 a 15, leve; variação de 16 a 25, moderada; e variação de 26 a 63, grave. O resultado obtido como mínimo foi considerado como ausência de ansiedade.

Para tratamento dos dados obtidos no estudo foi realizada análise descritiva e inferencial. A análise descritiva das variáveis foi realizada a partir da determinação das medidas de frequência (absoluta e relativa), seguida do teste de normalidade Shapiro-Francia. Dada a caracteristica não paramétrica dos dados, para a realização das medidas de associação foi calculado o coeficiente de correlação de Spearman e para o cálculo da diferença estatística foi utilizado o teste de Kruskal-Wallis. A análise foi considerada como significante para um valor-p menor ou igual a 0,05 e o intervalo de confiança de $95 \%$. Os dados foram processados com auxilio do software Stata 14.2.

O projeto foi aprovado pelo Comitê de Ética em Pesquisa da Faculdade Independente do Nordeste, sob número 3.101.464 em 22 de dezembro de 2018. Todos os concordantes em participar do estudo assinaram Termo de Consentimento após serem esclarecidos quanto à natureza e os objetivos da pesquisa.

\section{Resultados}

Participaram do estudo 366 estudantes universitários matriculados em cursos de ensino superior de uma faculdade privada da cidade de Vitória da Conquista, com idade variando de 18 a
57 anos, sendo a média de 26,8 anos ( \pm 7,1 anos).

Considerando-se o somatório do escores individuais de cada participante, 138 (37,7\%) deles tiveram classificação de grau mínimo, o que se configura em ausência de ansiedade, o restante da amostra (228 estudantes) apresentou algum grau de ansiedade. A média geral do escore da escala BAl foi de 14,13 ( \pm 12,90) o que resultou em um grau de ansiedade leve para a amostra (Tabela 1).

TABELA1 - Classificação dos estudantes entrevistados quanto ao grau de ansiedade, segundo somatório de escores individuais da escala BAI $(n=366)$

\begin{tabular}{ccc}
\hline Graus de ansiedade & $\Sigma^{*}$ & $\%$ \\
\hline Grau Minimo & 138 & 37,7 \\
Ansiedade Leve & 90 & 24,6 \\
Ansiedade Moderada & 75 & 20,5 \\
Ansiedade Grave & 63 & 17,2 \\
\hline
\end{tabular}

*Somatório dos escores individuais de cada participante.

A Tabela 2 mostra as frequências absolutas e relativas das variáveis sociodemográficas e culturais dos 366 universitários que participaram do estudo. Do total de estudantes que apresentaram algum grau de ansiedade (leve, moderado ou grave), a maioria eram mulheres (148 - 64,9\%), com idade entre 21 e 30 anos (135 - 59,2\%), solteiros (118 - 51,8\%), que moram com amigos (136 - 59,6\%), com filhos (176 - 77,2\%) e, realizando uma atividade de lazer, apenas (108 - 47,4\%). 
TABELA 2 - Distribuição das características sociodemográficas e comportamentais dos estudantes universitários segundo perfil de ansiedade calculado $(n=228)$

\begin{tabular}{|c|c|c|c|c|}
\hline \multirow{2}{*}{ Característica } & \multicolumn{3}{|c|}{ Graus de ansiedade } & \multirow{2}{*}{ Total } \\
\hline & $\begin{array}{l}\text { Leve } \\
(n=90)\end{array}$ & $\begin{array}{c}\text { Moderada (n } \\
=75)\end{array}$ & $\begin{array}{c}\text { Grave } \\
(n=63)\end{array}$ & \\
\hline Sexo & $n(\%)$ & n (\%) & n (\%) & n (\%) \\
\hline Masculino & $46(51,1)$ & $23(30,7)$ & $11(17,5)$ & $80(35,1)$ \\
\hline Feminino & $44(48,9)$ & $52(69,3)$ & $52(82,5)$ & $148(64.9)$ \\
\hline \multicolumn{5}{|l|}{ Faixa etária (em anos) } \\
\hline $18-20$ & $15(16,7)$ & $12(16,0)$ & $18(28,6)$ & $45(19.7)$ \\
\hline $21-30$ & $50(55,6)$ & $52(69,3)$ & $33(52,4)$ & $135(59,2)$ \\
\hline $31-40$ & $20(22,2)$ & $8(10,7)$ & $10(15,8)$ & $38(16,7)$ \\
\hline $41-50$ & $3(3,3)$ & $2(2,7)$ & $2(3,2)$ & $7(3,1)$ \\
\hline $51-60$ & $2(2,2)$ & $1(1,3)$ & - & $3(1,3)$ \\
\hline \multicolumn{5}{|l|}{ Estado Civil } \\
\hline Solteiro & $47(52,2)$ & $39(52,0)$ & $32(50,8)$ & $118(51,8)$ \\
\hline Namorando & $25(27,8)$ & $19(25.3)$ & $16(25,4)$ & $60(26,3)$ \\
\hline Noivo & $3(3,3)$ & $6(8,0)$ & $4(6,3)$ & $13(5.7)$ \\
\hline Casado & $15(16,7)$ & $11(14,7)$ & $11(17.5)$ & $37(16,2)$ \\
\hline \multicolumn{5}{|l|}{ Moradia } \\
\hline Sozinho & $13(14,5)$ & $11(14,7)$ & $8(12,7)$ & $32(14,0)$ \\
\hline Com amigos & $5561,1)$ & $46(61,3)$ & $35(55,6)$ & $136(59,6)$ \\
\hline Com a familia & $19(21,1)$ & $8(10,7)$ & $15(23,8)$ & $42(18,5)$ \\
\hline Em outra cidade & $3(3,3)$ & $10(13,3)$ & $5(7,9)$ & $18(7,9)$ \\
\hline \multicolumn{5}{|l|}{ Possui filhos } \\
\hline Sim & $69(76,7)$ & $57(76,0)$ & $50(79,3)$ & $176(77,2)$ \\
\hline Não & $21(23,3)$ & $18(24,0)$ & $13(20,6)$ & $52(22,8)$ \\
\hline \multicolumn{5}{|l|}{ Atividade de lazer } \\
\hline Nenhuma & $3(3,3)$ & $1(1,3)$ & $1(1,6)$ & $5(2,2)$ \\
\hline Uma atividade & $43(47,8)$ & $36(48,0)$ & $29(46,0)$ & $108(47,4)$ \\
\hline Duas atividades & $9(10,1)$ & $11(14,7)$ & $13(20,6)$ & $33(14,5)$ \\
\hline Três atividades & $21(23,3)$ & $14(18,7)$ & $9(14,3)$ & $44(19,3)$ \\
\hline Quatro atividades & $11(12,2)$ & $6(8,0)$ & $6(9,6)$ & $23(10,1)$ \\
\hline Cinco atividades & $3(3,3)$ & $4(5,3)$ & $4(6,3)$ & $1(4,8)$ \\
\hline Mais de cinco atividades & - & $3(4,0)$ & $1(1,6)$ & $4(1,7)$ \\
\hline
\end{tabular}

n: frequência absoluta. 
A Figura 1 mostra os graus de ansiedade dos participantes da pesquisa por área de conhecimento.

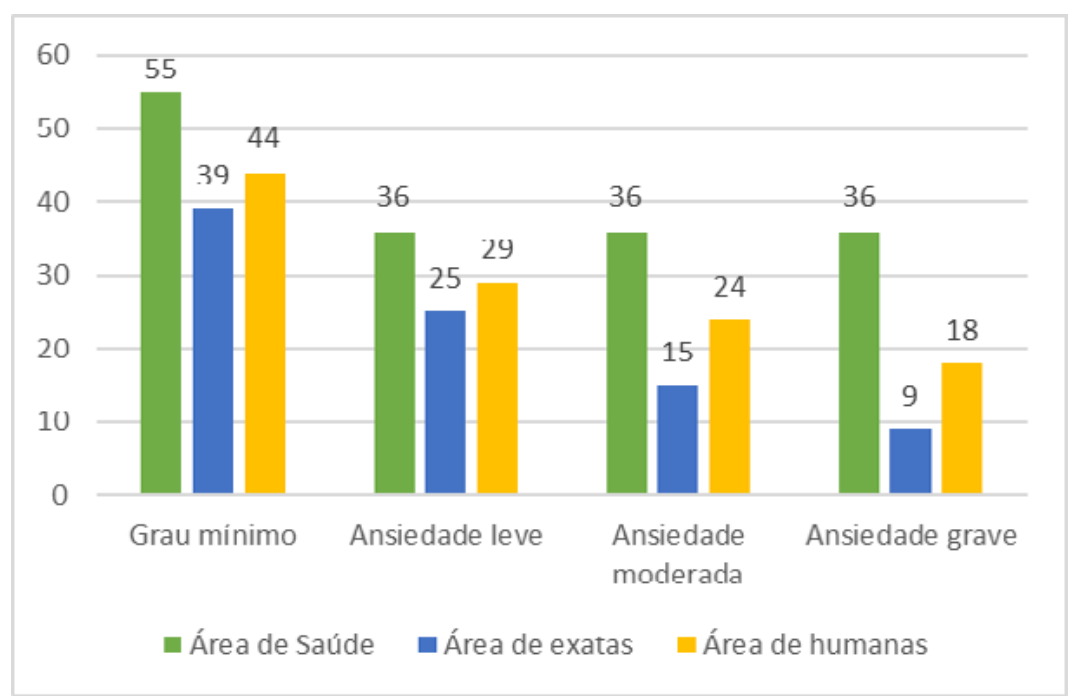

Figura 1 - Distribuição dos graus de ansiedade pela área do conhecimento $(n=366)$. Não foi encontrado diferença estatística significativa entre os grupos $(p=0,4069)$

Após cálculo do coeficiente de correlação de Spearman, pode-se observar que há uma associação significativa positiva entre o sexo e os graus de ansiedade e negativa entre a idade e os graus de ansiedade (Tabela 3 ).

TABELA 3 - Correlação entre as variáveis sociodemográficas e culturais e o grau de ansiedade dos estudantes universitários participantes da pesquisa

\begin{tabular}{lcc}
\hline \multicolumn{1}{c}{ Variáveis } & $\boldsymbol{\rho}$ & Valor $\mathbf{p}$ \\
\hline Sexo & 0,2494 & 0,0000 \\
Idade & $-0,1353$ & 0,0096 \\
Estado civil & 0,0033 & 0,9494 \\
Moradia & 0,0240 & 0,6476 \\
Filhos & $-0,0487$ & 0,3533 \\
Atividade de lazer & $-0,0258$ & 0,6227 \\
Curso & $-0,0355$ & 0,4981 \\
\hline
\end{tabular}

$\rho$ : coeficiente de correlação de Spearman.

\section{Discussão}

Diversas pesquisas acreditam que a ansiedade é o mal no tempo atual por considerarem que as sociedades modernas vivem em constantes mu- danças, isto é, aumento da tecnologia, transição econômica, transformações sociais, entre outras. Dessa forma, os sintomas acabam se tornando as queixas mais comuns encontradas nas demandas de saúde mental, a ponto de se destacar em relação aos demais transtornos mentais. Conforme a Organização Mundial da Saúde, o país com a maior porcentagem de pessoas com transtornos de ansiedade no mundo é o Brasil (9).

De acordo com Szpak e Kameg (10) a ansiedade é descrita como um estado de vários graus de inquietação ou desconforto, que atinge cerca de $12 \%$ da população universitária. Os transtornos de ansiedades são o problema mais comum de saúde mental visto entre os estudantes, com negativa influência em sua qualidade de vida e estando intimamente relacionada ao desempenho acadêmico, pois pode desencadear falta de interesse na aprendizagem e mau desempenho nos exames e nos trabalhos acadêmicos $(10,11)$.

A admissão na universidade compreende grandes mudanças, caracterizadas pela criação de relacionamentos novos, prováveis incertezas no que diz respeito à carreira escolhida, adaptação a novos ambientes, novos estilos de vida, aumento das responsabilidades, expectativas acadêmicas, entre outras. Assim explica-se o aparecimento da ansiedade e do estresse entre universitários (12-14). 
De acordo estudos de Medeiros et al. (15) há fatores externos que acarretam a ansiedade, mas as lembranças de situações anteriores, imagens, fantasias intimas, e o grau de intensidade desses é que vão definir o comportamento de ansiedade, podendo ser manifestada desde mínimos sintomas, e que vão aumentando gradativamente.

Em estudo realizado por Fawzy e Hamed (16), a alta prevalência de ansiedade entre estudantes está relacionada ao número de estressores acadêmicos e não acadêmicos, como sobrecarga de trabalho, falta de tempo de lazer, viver longe de suas familias com falta de apoio social, estados de relacionamento ruim com colegas e funcionários, preocupações financeiras e preocupações com o desempenho acadêmico. Segundo Guraya et al. (17), os três fatores mais estressantes que induziram a ansiedade grave em estudantes foram os exames, grandes quantidades de conteúdo do curso a ser aprendido e tempo insuficiente para revisar o que foi aprendido.

Por meio da aplicação da escala BAI de medição da ansiedade, principalmente por sua simplicidade, brevidade e capacidade de calcular a ansiedade geral (18), observamos que a maioria dos estudantes que apresentaram grau de ansiedade demonstrou tê-la em um grau leve, evidenciando a existência da ansiedade nos entrevistados, mesmo que de forma leve. Esse resultado está em concordância com os resultados da pesquisa de Marchi et al. (3).

Entretanto, a qualidade de vida dos estudantes e a formação da sua identidade podem ser abaladas de forma considerável com a presença da ansiedade em grau moderado e grave (3). Em nosso estudo, $37,7 \%$ apresentaram esses graus de ansiedade (20,5\% com grau de ansiedade moderada e $17,2 \%$ com grau grave). Nos estudos de Schotanus-Dijkstra et al. (19) 26\% dos entrevistados com transtorno de ansiedade relataram comprometimento grave, já nos estudos de Liu et al. (20) em 20\% dos alunos havia a presença de sintomas de ansiedade moderada a grave.

Niveis elevados de ansiedade não afetam apenas a saúde física dos alunos, mas também impede a atenção, a tomada de decisões, a capacidade cognitiva e afetam negativamente as condições no desempenho dos estudantes, provocando medo, dúvida e falta de expectativas (21).

De acordo pesquisa de Khoshhal et al. (22), a ansiedade durante um exame, é um conjunto de respostas que inclui preocupação excessiva, depressão e nervosismo em uma experiência de avaliação e resultado. De 20 a 35\% de todas as universidades há alunos que experimentam graus variados de ansiedade nas avaliações e até 40\% foram relatados na população mais jovem, sendo que os principais fatores que contribuíram para ansiedade são a carga horária extensiva e estudo na noite anterior ao exame, seguida por não estudar.

Pesquisadores e médicos especialistas em transtornos mentais identificam que, quando caracterizada em um grau intenso, a ansiedade pode gerar dores de cabeça e nas costas, arritmias cardiacas, determinados tipos de hipertensão arterial, transtornos digestivos, obesidade, doenças dermatológicas, dificuldade do sono, fadiga, entre muitas doenças, que até hoje não foram vistas como psicofisiológicas (9).

De acordo com Altintas, Yerdelen e Taskintuna (23), geralmente o transtorno de ansiedade aparece entre as idades de 13 e 24 anos, em mulheres solteiras e pode causar sérios distúrbios no desempenho vocacional, social e acadêmico, no qual os distúrbios de personalidade e fatores genéticose biológicos, desempenham um papel na etiologia.

Estudos realizados com universitários demonstraram haver uma maior prevalência de ansiedade em mulheres, apontando estas serem mais susceptiveis ao desenvolvimento e à manifestação de diferentes subtipos de ansiedade principalmente em determinados períodos do ciclo hormonal feminino, durante os quais as concentrações de estrogênio e de progesterona encontram-se mais baixas ou apresentam-se mais variáveis $(2,14,24)$. Nosso estudo também identificou que dos participantes que apresentaram algum grau de ansiedade a maioria era do sexo feminino, inclusive foi observado uma forte correlação entre o sexo e os graus de ansiedade.

Outro fator que pode esclarecer o predomínio de níveis mais elevados de ansiedade no sexo feminino é o fato de que a mulher, na sociedade atual, ainda tem que vencer mais bloqueios 
para adentrar em uma carreira que permita uma melhor independência econômica e social, confrontando maiores batalhas que os homens e, por isso, tende a reagir com maior grau de ansiedade em condições de pressão psicológica (2).

A idade dos participantes foi também um fator fundamental na avaliação do grau de ansiedade, pois encontrou-se uma considerável associação de idade mais jovem que apresentaram ansiedade, o que explica o fato desses alunos criarem expectativas sobre sua vida acadêmica e seu futuro profissional, comparado com os alunos de idade maior. Contudo, a ansiedade também pode estar relacionada com anos de estudo, indicando-a em alunos mais experientes (13).

Em contrariedade com o nosso estudo, os autores Dalky e Gharaibeh (25) observaram que 29\% dos participantes da pesquisa apresentaram ansiedade severa e 18,6\% moderada. Observaram também uma correlação com a idade, no qual os escores de ansiedade foram significativamente maiores entre os alunos mais experientes (20 anos e acima). Uma razão para isso é que, à medida que os alunos aumentam a idade, tornam-se mais maduros, com mais responsabilidades e desafios.

A ansiedade é comum entre os jovens, pois a idade adulta jovem é um período de desenvolvimento crítico e de transição, na qual seus sintomas podem ser negligenciados. Pode estar relacionada a um conjunto de fatores ambientais e psicológicos, que prepara o indivíduo para circunstâncias de risco, mas que, juntamente com o medo, envolvem aspectos afetivos, comportamentais, fisiológicos, neurológicos e alterações psicossociais, que podem ser suscetiveis para o desenvolvimento de transtornos de ansiedade (11, 26). Achados de Carvalho et al. (2) têm demonstrado que há elevados escores de ansiedade em amostras de individuos mais jovens. Isso também foi observado em nosso estudo, além de confirmado pela correlação negativa entre a idade e os graus de ansiedade calculada no estudo: quanto mais jovem, maior o escore de ansiedade obtido ( $\rho=-0,1353, p$-valor $=0,0096$ ).

Nosso estudo demostrou que houve uma quantidade maior de participantes que praticavam apenas uma atividade de lazer que possuíam algum grau de ansiedade (leve, moderado ou grave) do que comparado aos alunos que praticavam três atividades de lazer. O estilo de vida e a prática de atividade de lazer, como a atividade física, apresentam-se como meios efetivos para controlar dos niveis de ansiedade. Há evidências de que o exercício físico pode influenciar a evolução dos distúrbios (20).

Segundo a pesquisa de Fernani et al. (27) que relacionou o esporte à ansiedade e à qualidade de vida, os atletas estão menos ansiosos do que as pessoas sedentárias, mostrando que a atividade física contribui para a o bem-estar emocional. Além disso, eles também relacionaram o nivel de ansiedade ao exercício físico e descobriram que, independentemente do nivel de ansiedade, os jovens respondem de maneira semelhante aos exercícios de diferentes intensidades, com diminuição nivel de ansiedade.

Dado que a maioria dos participantes desta pesquisa que apresentaram algum grau de ansiedade, moram com amigos, entende-se que eles não são naturais da cidade onde a faculdade está situada. Assim, o fato de morar distante da família pode ser visto como um fator de ansiedade, devido à necessidade de deixar a casa dos pais e começar uma vida desapegada das facilidades desse lar, a ansiedade acaba se tornando parte indispensável do processo de mudança, pois o estudante passa a ter preocupações que anteriormente não tinha (4). Segundo Othman et al. (13) deslocação entre casa e faculdade também foi um forte preditor para determinar o nivel moderado e grave na pontuação de ansiedade.

Com base nos resultados de pesquisa realizada por Beiter et al. (28), alunos que vivem fora de casa são classificados nos niveis mais altos de ansiedade, fator mais provável de influenciar esse resultado é o fato deles apresentem um estresse com relação a pagamentos de contas a cada mês, planejamentos, preparação de refeições e problemas que podem surgir no seu domicílio.

Segundo Lantyer et al. (14), estudantes universitários, particularmente da área da saúde, exibem niveis elevados de ansiedade. Em nossa pesquisa pode-se observar o mesmo cenário que, indepen- 
dentemente do grau de ansiedade apresentado (leve, moderado ou grave), os estudantes dos cursos da área de saúde apresentaram as maiores frequências, contudo, não foi observado diferença estatística significativa entre os grupos da amostra.

Na pesquisa de Sun et al. (21), os resultados demonstraram que os fatores que contribuíram para ansiedade em alunos da área de saúde incluiam falta de conhecimento; medo de cometer erros; primeira aplicação das habilidades; e mudança da condição dos pacientes, comunicação com pacientes, familiares e outros profissionais de saúde, juntamente com a avaliação dos preceptores.

A identificação de fatores associados ao grau de ansiedade em jovens universitários nos permite pensar em estratégias de intervenção, tornando nosso estudo de grande relevância. Os sintomas provocados pela ansiedade geram grande impacto na saúde dos alunos, pois normalmente só procuram assistência médica em sintomas físicos, e desconhecem que eles podem estar relacionados a algum transtorno de saúde mental. Métodos para detecção devem ser incluidos nos cuidados da saúde de rotina, para não impedir os pacientes de procurarem ajuda. Mais estudos nas populações universitárias são necessários para conquistar um melhor entendimento sobre a ansiedade e suas consequências.

Este estudo implica que há uma necessidade crescente de que faculdades considerem implementar estratégias para cuidar da saúde mental dos seus alunos, de forma a oferecer serviços de atenção, para que esses sejam atendidos tanto em suas necessidades pedagógicas quanto emocionais. Desta forma, promoverá uma formação mais humanizada, que beneficie a saúde mental dos universitários, para que eles estejam tecnicamente e emocionalmente preparados para o mercado de trabalho.

\section{Notas}

\section{Apoio financeiro}

Este estudo não recebeu apoio financeiro de fontes externas.

\section{Declaração de conflito de interesses}

Os autores declaram não haver conflitos de interesses relevantes ao conteúdo deste estudo.

\section{Contribuições dos autores}

Todos os autores fizeram contribuições substanciais para concepção, ou delineamento, ou aquisição, ou análise ou interpretação de dados; e redação do trabalho ou revisão crítica; e aprovação final da versão para publicação.

\section{Referências}

1. Sousa JM de, Moreira CA, Telles-Correia D. Anxiety, depression and academic performance: a study amongst portuguese medical students versus non-medical students. Acta Med Port. 2018;31(9):454-62. https://doi. org/10.20344/amp.9996

2. Carvalho EA de, Bertolini SMMG, Milani RG, Martins $M C$. Índice de ansiedade em universitários ingressantes e concluintes de uma instituição de ensino superior. Ciênc Cuid Saúde.2015; 14(3):1290-98. https://doi. org/10.4025/cienccuidsaude.v14i3.23594

3. Marchi KC, Bárbaro AM, Miasso Al, Tirapelli CR. Ansiedade e consumo de ansiolíticos entre estudantes de enfermagem de uma universidade pública. Rev Eletrônica Enferm. 2013; 15(3):731-39. https://doi.org/10.5216/ree.v15i3.18924

4. Carvalho MRF de, Rodrigues ET, Golzio AMF de O. Intervenções no uso prolongado de benzodiazepínicos: uma revisão. RSC online, 2016;5(2):55-64.

5. Al-Sayed AA, Al-Rashoudi AH, Al-Eisa AA, Addar AM, Al-Hargan AH, Al-Jerian AA, Al-Omair AA, Al-Sheddi Al, Al-Nowaiser HI, Al-Kathiri OA, Al-Hassan AH. Sedative drug use among King Saud University medical students: a cross-sectional sampling study. Depress Res Treat. 2014: 378738:doi: http://dx.doi.org/10.1155/2014/378738

6. Gorday JY, Rogers ML, Joiner TE. Examining characteristics of worry in relation to depression, anxiety, and suicidal ideation and attempts. J Psychiatr Res. 2018;107:97-103. https://doi.org/10.1016/i.jpsychires.2018.10.004

7. Nunes BS, Bastos FM. Efeitos colaterais atribuidos ao uso indevido e prolongado de benzodiazepínicos. Saúde \& ciência em ação. 2016; 3(01):71-82.

8. Kapil V, Green JL, Lait CL, Wood DM, Dargan PI. Misuse of benzodiazepines and Z-drugs in the UK. Br J Psychiatry. 2014; 205(05): 407-08. https://doi.org/10.1192/ bjp.bp.114.149252

9. Ximenes CR, Neves GMB. Transtornos de ansiedade: importância da avaliação psicológica no diagnóstico e tratamento. Revista Uni. 2018; 18(1/2): 121-38. 
10. Szpak JL, Kameg KM. Simulation decreases nursing student anxiety prior to communication with mentally ill patients. Clin Simul Nurs. 2013; 9:13-19. https://doi.org/10.1016/j.ecns.2011.07.003

11. Cardozo MQ, Gomes KM, Fan L G, Soratto MT. Fatores associados à ocorrência de ansiedade dos acadêmicos de Biomedicina. Saúde e Pesq. 2016; 9(2): 251-62. https://doi.org/10.17765/1983-1870.2016vgn2p251-262

12. Chiu HT, Yee LTS, Kwan JLY, Cheung RYM, Hou WK. Interactive association between negative emotion regulation and savoring is linked to anxiety symptoms among college students. J Am Coll Health. 2019; p.1-8. https://doi.org/10.1080/07448481.2019.1580712

13. Othman N, Ahmad F, Morr CE, Ritvo P. Perceived impact of contextual determinants on depression, anxiety and stress: a survey with university students. Int J Ment HealthSyst. 2019; 13(1):1-9. https://doi.org/10.1186/s13033-019-0275-x

14. Lantyer AS, Varanda CC, Souza FG, Padovani RC, Viana MB. Ansiedade e qualidade de vida entre estudantes universitários ingressantes: avaliação e intervenção. Rev Bras Ter Cogn. 2016;2:4-19. https://doi.org/10.31505/rbtcc.v18i2.880

15. Medeiros L de A, Ramiro FMS, Lima CAA, Souza LM de A, Fortes TMV, Groppo FC. Avaliação do grau de ansiedade dos pacientes antes de cirurgias orais menores. Rev Odontol UNESP. 2013:5(42):357-63. https:// doi.org/10.1590/S1807-25772013000500007

16. Fawzy M, Hamed SA. Prevalence of psychological stress, depression and anxiety among medical students in Egypt. Psychiatry Res. 2017 Sep;255:186-194. https:// doi.org/10.1016/j.psychres.2017.05.027

17. Guraya SY, Guraya SS, Habib F, AlQuiliti KW, Khoshhal $\mathrm{KI}$. Medical students' perception of test anxiety triggered by different assessment modalities. Medical Teach. 2018 Sep;40(sup1):S49-S55. https://doi.or$\mathrm{g} / 10.1080 / 0142159 \times .2018 .1465178$

18. Lemos MF, Lemos Neto SV, Barrucand, L., Verçosa, $\mathrm{N}$, Tibirica, E. A informação no pré-operatório reduz a ansiedade pré-operatória em pacientes com câncer submetidos à cirurgia: utilidade do Inventário Beck de Ansiedade. Rev Bras Anestesiol. 2019; 69(1):1-6. https://doi.org/10.1016/j.bjan.2018.07.003

19. Schotanus-Dijkstra M, Keyes CLM, Graaf R de, Have M. Recovery from mood and anxiety disorders: the influence of positive mental health. J Affect Disord.2019; 107-113. https://doi.org/10.1016/j.jad.2019.04.051

20. Liu C, Wang L, Qi R, Wang W, Jia S, Shang D, Shao Y, Yu M, Zhu X, Yan S, Chang Q, Zhao Y. Prevalence and associated factors of depression and anxiety among doctoral students: the mediating effect of mentoring relationships on the association between research self-efficacy and depression/ anxiety. Psychol Res Behav Manag.2019; 12:195-208. https://doi.org/10.2147/PRBM.S195131
21. Sun FK, Long A, Tseng YS, Huang HM, You JH, Chiang CY Undergraduate student nurses' lived experiences of anxiety during their first clinical practicum: A phenomenological study. Nurse Education Today. 2016; 37:21-26. https://doi.org/10.1016/i.nedt.2015.11.001

22. Khoshhal KI, Khairy GA, Guraya SY, Guraya SS. Exam anxiety in the undergraduate medical students of Taibah University. Med Teach. 2017 Apr;39(sup1):S22-S26. https://doi.org/10.1080/0142159X.2016.1254749

23. Altintas E, Yerdelen VD, Taskintuna N. Social anxiety level in adult patients with epilepsy and their firstdegree cohabiting relatives. J Neuropsychiatry Clin Neurosci. 2015; 27(4):339-44. https://doi.org/10.1176/ appi.neuropsych.15030061

24. Vasconcelos TC de, Dias BRT, Andrade LR, Melo GF. Barbosa L, Souza E. Prevalência de sintomas de ansiedade e depressão em estudantes de medicina. Rev Bras Educ Med. 2015; 39(1):35-42. https://doi.org/10.1590/1981-52712015V39n1e00042014

25. Dalky HF, Gharaibeh A. Depression, anxiety, and stress among college students in Jordan and their need for mental health services. Nursing Forum, 2018; 54(2):205-12. https://doi.org/10.1111/nuf.12316

26. Mondin TC, Konradt CE, Cardoso TA, Quevedo LA, Jansen K, Mattos LD, Pinheiro RT, Silva, RA. Anxiety disorders in young people: a population-based study. Rev Bras Psiquiatr. 2013 Dez; 35(4):347-52. https://doi. org/10.1590/1516-4446-2013-1155

27. Fernani DCGL, Cortez GM, Santos IA, Pacagnelli FL, Massetti T, Silva TD, Papa, DCR Moraes ÍAP, Monteiro CBM, Prado MTA. Anthropometric profile, physical activity level, degree of anxiety, and posture in college students. Fisioter. Pesq. 2017;24(2):191-97. https://doi.org/10.1590/1809-2950/17160324022017

28. Beiter R, Nash R, McCrady M, Rhoades D, Linscomb M, Clarahan M, Sammut S. The prevalence and correlates of depression, anxiety, and stress in a sample of college students. J Affect Disord. 2015 Mar 1;173:90-6. https://doi.org/10.1016/j.jad.2014.10.054

\section{Endereço para correspondência}

Rafael Luiz de Araújo Rodrigues

Faculdade Independiente do Nordeste

Av. Luiz Eduardo Magalhães, 1305

45028440

Vitoria da Conquista, BA, Brasil 